\title{
Measurements of density dependent intensity ratios of extreme ultraviolet line emission from Fe X, XI, and XII
}

\author{
Erina Shimizu ${ }^{1}$, Safdar Ali $^{1}$, Takashi Tsuda ${ }^{1}$, Hiroyuki A. Sakaue ${ }^{2}$, Daiji Kato ${ }^{2,3}$, Izumi Murakami ${ }^{2}, 3$, \\ Hirohisa Hara $^{4,5}$, Tetsuya Watanabe ${ }^{4,5}$, and Nobuyuki Nakamura ${ }^{1}$ \\ ${ }^{1}$ Institute for Laser Science, The University of Electro-Communications, Tokyo 182-8585, Japan \\ e-mail: safdaruetian@gmail.com \\ 2 National Institute for Fusion Science, Gifu 509-5292, Japan \\ 3 Department of Fusion Science, SOKENDAI, Gifu 509-5292, Japan \\ ${ }^{4}$ National Astronomical Observatory of Japan, Tokyo 181-8588, Japan \\ 5 Department of Astronomical Science, SOKENDAI, Tokyo 181-8588, Japan
}

Received 6 December 2016 / Accepted 25 March 2017

\begin{abstract}
We report high-resolution density dependent intensity ratio measurements for middle charge states of iron in the extreme ultraviolet (EUV) spectral wavelength range of 160-200 $⿱$. The measurements were performed at the Tokyo EBIT laboratory by employing a flat-field grazing incidence spectrometer installed on a low energy compact electron beam ion trap. The intensity ratios for several line pairs stemming from Fe X, Fe XI and Fe XII were extracted from spectra collected at the electron beam energies of 340 and $400 \mathrm{eV}$ by varying the beam current between 7.5 and $12 \mathrm{~mA}$ at each energy. In addition, the effective electron densities were obtained experimentally by imaging the electron beam profile and ion cloud size with a pinhole camera and visible spectrometer, respectively. In this paper, the experimental results are compared with previous data from the literature and with the present calculations performed using a collisional-radiative model. Our experimental results show a rather good agreement with the calculations and previous reported results.
\end{abstract}

Key words. ultraviolet: planetary systems - Sun: corona - methods: laboratory: atomic - techniques: spectroscopic atomic processes - atomic data

\section{Introduction}

Spectroscopy is an important tool to study physical conditions and processes taking place in astrophysical sources. Almost all the information from astrophysics comes by means of spectroscopy (Dalgarno \& Layzer 1987). By studying the emission line spectra one can obtain basic plasma properties such as electron densities, electron temperatures, elemental abundances, and equilibrium conditions, to name a few (Dwivedi 1993; Mason et al. 1997). The electron density is one of the most important and fundamental parameters used to describe thermal plasma (Kurth et al. 2015; Liang et al. 2009a). It plays a vital role in the construction of theoretical models and codes used for understanding the chemical evolution of the stellar and galactic composition, structure and properties of, for example, flares, galaxies, stellar corona, and nebulae (Beiersdorfer et al. 2014a; Young et al. 2009; Shestov et al. 2009; Gćdel \& Nazé 2009; Wamsteker et al. 2006). The electron density can be determined by taking relative intensities of the two spectral lines that are sensitive to the electron density (Young et al. 2009). Highly accurate density dependent intensity ratio measurements are therefore required for interpreting and modelling astrophysical plasmas.

The extreme ultraviolet (EUV) spectral wavelength range contains most of the emission lines originating from highly charged ions (HCIs) of low to middle Z elements (Liang et al. 2009a). In particular, the line emission in the temperature range of 1-3 MK (coronal temperature) is mainly due to transitions from the middle charge states of iron. Many of these transitions provide excellent density diagnostics for coronal plasmas below $300 \AA$ (Beiersdorfer et al. 2012; Young et al. 2009). The EUV Imaging Spectrometer (EIS) on board the Hinode satellite (Culhane et al. 2007) record high resolution spectra in this wavelength band (below $300 \AA$ ) and thus provides motivation to perform laboratory observations for density diagnostics (Ali et al. 2015). The laboratory results of the EUV emission from Fe X, XI, and XII presented here, are of particular interest to the data collected by the Hinode mission, where they can be used to extract density information from data obtained by the EIS (Del Zanna et al. 2010; Del Zanna 2012).

The recent launch of advanced and high-resolution space based EUV spectrometers makes it possible to record emission spectra from hot astrophysical plasmas in unprecedented detail. These include the Atmospheric Imaging Assembly (AIA) on board the Solar Dynamics Observatory (SDO; Lemen et al. 2012), the Cosmic Hot Interstellar Plasma Spectrometer (CHIPS; Hurwitz et al. 2005), the Coronal Diagnostic Spectrometer (CDS; Harrison et al. 1995) on board the Solar and Heliospheric Observatory (SOHO) satellite, the EUV Imaging Spectrometer (EIS; Brown et al. 2008) onboard on solar observatory Hinode (Solar-B), the Solar EUV Rocket Telescope and Spectrograph (SERTS; Neupert et al. 1992), the LowEnergy Transmission Grating Spectrometer (LETGS) on the Chandra X-ray observatory (Mewe et al. 2001) amongst others. The high quality spectroscopic data detected with these space 
based observatories provide challenging opportunities for the ground based instruments to reproduce and verify these observations for the implementation in future research and plasma modelling.

So far, the EUV emission spectra of highly charged iron, notably Fe VIII through Fe XV have been observed from astrophysical observations and identified with the help of experimental data (Thomas \& Neupert 1994; Young et al. 2007; Yamamotto et al. 2008; Lepson et al. 2008; Del Zanna 2012; Brickhouse et al. 1995; Schmitt et al. 1996; Feldman et al. 2008; Beiersdorfer et al. 2012, 2014a,b). However, density dependent line ratios data for these ions and in particular Fe X to XII, is still not very well known. To the best of our knowledge, the only results reported for the line intensity ratios of Fe X to XII from an EBIT are by Liang et al. (2009a,b) using the FLASH-EBIT in Heidelberg. However, discrepancies exist in their observations and simulated results which demand more detailed studies on these ions as pointed out by the authors. They have not obtained any direct information for the ion cloud and electron beam size in their experiments and used theoretical electron beam width (Herrmann 1958) to obtain the electron density. They therefore suggested to perform more detailed studies for these ions with EBITs by setting various operating conditions. Secondly, some of the transitions belonging to charge states Fe X, Fe XI and Fe XII were blended in their recorded spectra and imposed restriction to extract line intensity ratios corresponding to each single transition. We therefore decided to perform measurements on these ions with high-resolution and to derive electron beam and ion cloud overlap experimentally by direct observations.

In this paper, we present high-resolution density dependent intensity ratios for EUV radiation emitted from highly charged Fe X, XI and XII ions in the wavelength range of 160-200 A, recorded with a grazing-incidence flat-field grating spectrometer (Nakamura et al. 2008). Rather than estimating electron density from the theoretical electron beam width as reported previously, here we obtained it experimentally by directly imaging electron beam and observing spatial distribution of the trapped ions. To compare and estimate the measured intensity ratios, we have performed collisional-radiative model calculation using the HULLAC code. The experimentally obtained density dependent intensity ratios for various spectral lines of Fe X, XI and XII are presented and compared with present calculations and previous studies.

\section{Experiment and data analysis}

The EUV emission measurements were performed using a compact electron beam ion trap called CoBIT at the Tokyo EBIT laboratory, The University of Electro-communications Tokyo, Japan (Sakaue et al. 2010). The schematic of the experimental set up is shown in Fig. 1. This EBIT was designed as a low energy, low-density spectroscopic facility to study HCIs of astrophysical interest. Here, we give brief overview of the experimental methods and parameters, more detail about the device and measurements procedure can be found in our previous papers (Nakamura et al. 2008, 2011). Briefly, the CoBIT mainly composed of electron gun, ion trapping region and electron collector. The ion-trapping region consists of three successive drift tubes surrounded by a superconducting magnet with a maximum central magnetic field of $0.2 \mathrm{~T}$. This field is necessary to compress the electron beam with a typical density of $10^{11} \mathrm{~cm}^{-3}$. It also helps to guide the beam through the drift tube assembly. The ions are trapped axially by the outer drift tubes (both are on high potential compared to the middle one, thus provide a well

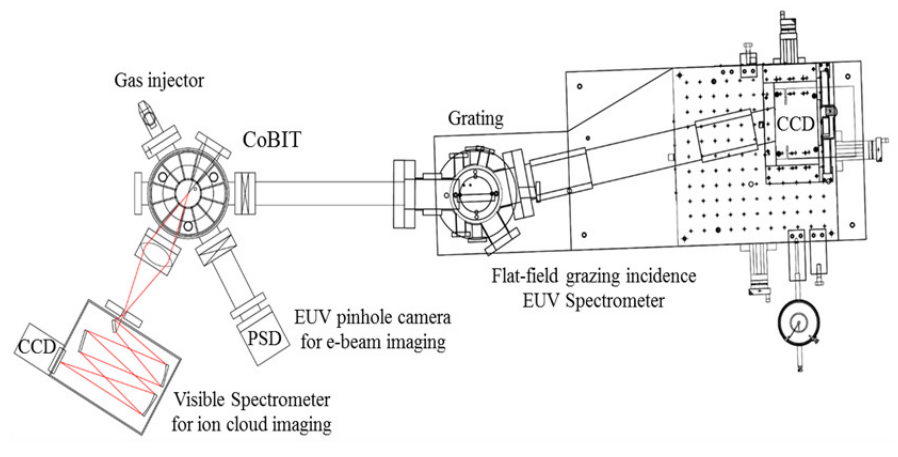

Fig. 1. Schematic drawing of the experimental setup used in the present measurements.

shape potential to the ions) and radially by the applied magnetic field and space charge produced by the electron beam. HCIs are produced through successive collisions of the electron with the injected species and trapped in the centre of the trap.

To produce ionization balance of iron, a molecular gas of organometallic compound, ferrocene $\left(\mathrm{Fe}\left(\mathrm{C}_{5} \mathrm{H}_{5}\right)_{2}\right)$, was injected into the trapping region of CoBIT via a gas injection system, while keeping the CoBIT pressure below $10^{-10}$ Torr. The desired charge states were produced by selecting an appropriate electron beam energy and held in the centre of trap by applying a $30 \mathrm{~V}$ potential on the outer drift tubes. The present measurements were performed at two different electron beam energies: 340 and $400 \mathrm{eV}$. The EUV emission from the trapped ions was observed by employing a high-resolution grazing incidence flatfield grating spectrometer (Sakaue et al. 2010). To obtain a high order of dispersion, we used a concave grating with large radius of curvature (Hitachi 001-0660). The average groove number is same as that of the flat-field grazing incidence spectrometer grating used in our previous studies (Nakamura et al. 2011), however, the larger radius of curvature $(13450 \mathrm{~mm})$ and the larger distance from the grating to the focal plane $(563.2 \mathrm{~mm})$ makes the dispersion on the focal plane higher as $2.6 \AA / \mathrm{mm}$ (Ali et al. 2015). Since an EBIT represents a thin line shape source, in the present setup, we therefore used no entrance slit, that is, the spectrometer was used in a slitless configuration. The spectral resolution in the present measurements is very much improved $(0.4 \AA)$ compared to our earlier reported results $(0.8 \AA$; Nakamura et al. 2011).

The spectroscopic data was recorded with an automatic data acquisition system and stored counts and channel numbers to the data file in a lab computer. The electron density inside the trap was varied by varying the beam current between 7.5 and $12 \mathrm{~mA}$ at each energy mentioned above. The counts corresponding to each current and beam energy was recorded for an exposure time of $30 \mathrm{~min}$. To avoid accumulation of impurities from heavy elements such as $\mathrm{W}$ and $\mathrm{Ba}$, the trap was filled and emptied periodically. Indeed, the recorded spectra do not contain any line from these elements and thus contamination or line blending from these elements is overruled in the present measurements. Wavelength calibration of the measured spectra were performed using several well known Fe lines [Fe XIII (203.826 ̊), Fe XII (186.887, $195.119 \AA$ A), Fe XI (180.401 $)$, Fe X (174.531 A), Fe IX (171.073 A)] by fitting the calibration curve with a third degree polynomial.

The uneven population of the magnetic sub-levels in HCIs excited with a unidirectional monenergistic electron beam leads to the emission of photons having an-isotropic angular distribution and polarization characteristics (Beiersdorfer et al. 1996; 
$\mathrm{Hu}$ et al. 2014a). The measured intensity of the emitted radiation from these ions strongly depends on the angle of observation relative to the beam axis. There have been several studies reported in the literature for the polarization of radiation emitted from ions trapped in an EBIT (Henderson et al. 1990; Beiersdorfer et al. 1996; Taḱacs et al. 1996; Gu et al. 1999; Liang et al. 2009a; Hu et al. 2014b; Shah et al. 2015). In the present study, we measured EUV spectra using CoBIT at $90^{\circ}$ with respect to the electron beam, the polarization of the emitted line radiation may therefore effect the resulting line intensities as discussed by (Liang et al. 2009a; Beiersdorfer et al. 1996; Henderson et al. 1990). Liang et al. (2009a) estimated polarization effect for the transitions in Fe VII-Fe XIV and found that for most of the observed lines, the contribution is less than $10 \%$ to the resultant intensity. We also estimated the contribution of polarization to the $\mathrm{Fe} \mathrm{XV}$ transitions in our earlier publication (Nakamura et al. 2011) and found these effects as negligible. We thus made no corrections for the polarization in our results.

In most of the previous measurements performed on EBITs, theoretically estimated electron-ion overlap factor have been used to derive electron densities and thus corresponding density sensitive line ratios (Liang et al. 2009a,b; Chen et al. 2004; Yamamotto et al. 2008). This resulted in uncertainties in the measured electron density values. In the present measurements, we have measured overlap factor by directly imaging the electron beam and spatial distribution of ions. A pinhole camera was used to obtain the electron beam profile as discussed in Nakamura et al. (2011). Briefly, the pinhole camera set up consisted of a $0.2 \mathrm{~mm}$ wide slit placed at a distance of $30 \mathrm{~mm}$ from the electron beam and a position sensitive detector with a microchannel plate placed at $320 \mathrm{~mm}$ from the slit. With this arrangement we have obtained spatial distribution of the EUV emission with a magnification factor of $\sim 11$. The EUV emission distribution represents the electron beam distribution since the lifetime of EUV transitions is of the order of $10^{-10} \mathrm{~s}$. A typical EUV image distribution obtained with the pinhole camera at electron beam energy of $340 \mathrm{eV}$ with beam current of $10 \mathrm{~mA}$ is shown in Fig. 2a. By fitting the Gaussian function to this peak we obtained the size of the electron beam (FWHM) with a value of $264 \mu \mathrm{m}$. The asymmetric shape of the image could be due to the misalignment between the magnetic field and the trap electrodes, although we are not sure at present.

The ion cloud distribution was observed with a CzernyTurner visible spectrometer. The emission from trapped ions was focused onto the entrance slit of the visible spectrometer with a biconvex lens having unity magnification. The diffracted light was detected by using a backilluminated CCD to record image from visible transitions. The slit was fully opened $(\sim 2 \mathrm{~mm})$ during ion cloud imaging for preventing the focused image being interrupted. The visible emission distribution is considered to represents the ion cloud distribution since the visible transition in HCIs has long lifetime - of the order of $10^{-3} \mathrm{~s}$. It is important to mention here that the spectrometer can record spectral image from one charge state alone (no blending) at a particular wavelength. As an example we show an image of Fe XI at $789.18 \AA$ $\left(3 s^{2} 3 p^{4}{ }^{3} P_{2}-3 s^{2} 3 p^{4}{ }^{3} P_{1}\right)$ in Fig. $2 b$ recorded at $340 \mathrm{eV}$ with electron beam current of $10 \mathrm{~mA}$. By fitting the Gaussian function to this peak, we obtained the size of the ion cloud (FWHM) with a value of $834 \mu \mathrm{m}$.

\section{Electron densities}

In an EBIT, the electron density $n_{\mathrm{e}}$ is determined by the electron beam current $\left(I_{\mathrm{e}}\right)$, velocity $\left(v_{\mathrm{e}}\right)$ and size. When the electron beam
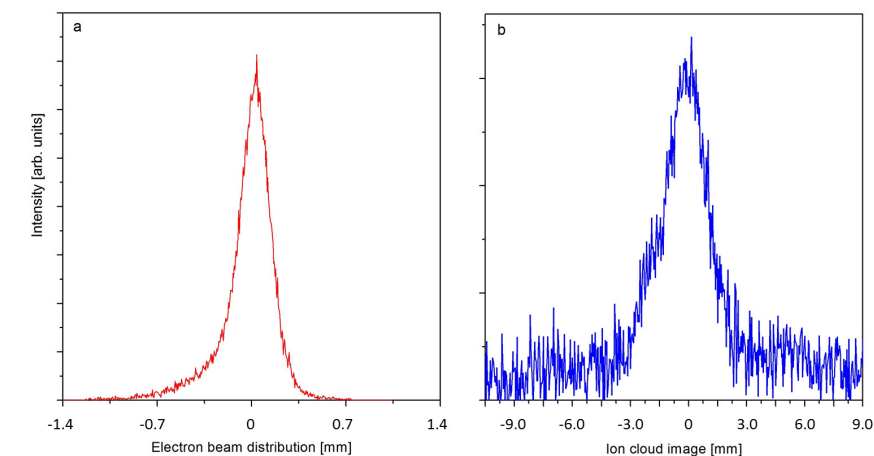

Fig. 2. a) Electron beam distribution recorded with a pinhole camera at beam energy of $340 \mathrm{eV}$ and a beam current of $10 \mathrm{~mA}$. b) Ion cloud image distribution observed with a visible spectrometer for Fe XI transition $3 s^{2} 3 p^{4}{ }^{3} \mathrm{P}_{2}-3 s^{2} 3 p^{4}{ }^{3} \mathrm{P}_{1}$ at the wavelength of $789.18 \AA$.

is assumed to be a uniform cylindrical beam with a radius $r_{\mathrm{e}}$, the density is determined by

$n_{\mathrm{e}}^{\mathrm{uni}}=\frac{I_{\mathrm{e}}}{\pi r_{\mathrm{e}}^{2} e v_{\mathrm{e}}}$

However, in general, the electron beam in an EBIT is considered to have a Gaussian distribution. When the FWHM of the distribution is $\Gamma_{\mathrm{e}}$, the averaged density for the radius $r_{80}$ containing $80 \%$ of the beam electrons is determined by (Herrmann 1958)

$n_{\mathrm{e}}^{\mathrm{g}}=\frac{0.8 \times I_{\mathrm{e}}}{\pi r_{80}^{2} e v_{\mathrm{e}}}=\frac{\alpha I_{\mathrm{e}}}{\pi \Gamma_{\mathrm{e}}^{2} e v_{\mathrm{e}}}$,

where $\alpha$ is a constant $(\alpha \sim 1.38)$. This is the intrinsic density of the electron beam, and referred to as the geometrical density hereafter. On the other hand, the ions trapped in an EBIT are also consider to have a Gaussian distribution. Thus, the effective electron density $n_{\mathrm{e}}^{\text {eff }}$, which governs the collision frequency, should be considered by taking the overlap between the electron and ion distributions into account. When the FWHM of the ion distribution is $\Gamma_{\mathrm{i}}$, the averaged effective density can be derived by

$n_{\mathrm{e}}^{\mathrm{eff}}=\frac{4 \ln (2) I_{\mathrm{e}}}{\pi\left(\Gamma_{\mathrm{e}}^{2}+\Gamma_{\mathrm{i}}^{2}\right) e v_{\mathrm{e}}}$.

When the electrons and ions are assumed to have the same distribution, that is, the same FWHM $\Gamma$, the effective density becomes $2 \ln (2) I_{\mathrm{e}} / \pi \Gamma^{2} e v_{\mathrm{e}}$, which is almost equivalent to the geometrical density $n_{\mathrm{e}}^{\mathrm{g}}$. In general, the trapped ions have a wider distribution compared with the electron beam as reported in several previous studies (Gillaspy et al. 1995; Chen et al. 2004; Silver et al. 2000; Liang et al. 2009b; Nakamura et al. 2011). The effective density $n_{\mathrm{e}}^{\mathrm{eff}}$ therefore becomes smaller than the geometrical density $n_{\mathrm{e}}^{\mathrm{g}}$.

In this work we have obtained the effective electron densities by measuring the size of the electron beam and ion cloud for each charge state and then by using these values in Eq. (3). For example, by using the values of beam current, electron beam and ion cloud size $\left(I_{\mathrm{e}}=10 \mathrm{~mA}, \Gamma_{\mathrm{e}}=264 \mu \mathrm{m}, \Gamma_{\mathrm{i}}=834 \mu \mathrm{m}\right)$ in Eq. (3), we obtained the electron density $6.6 \times 10^{9} \mathrm{~cm}^{-3}$ for Fe XI. By adopting the same procedure we extracted electron densities for other $\mathrm{Fe}$ ions as given in Fig. 4 (to be discussed later). By assuming equal size of the electron beam and ion cloud, we also estimated geometrical electron density with a value of $3.6 \times 10^{10} \mathrm{~cm}^{-3}$, which is about a factor of five higher 


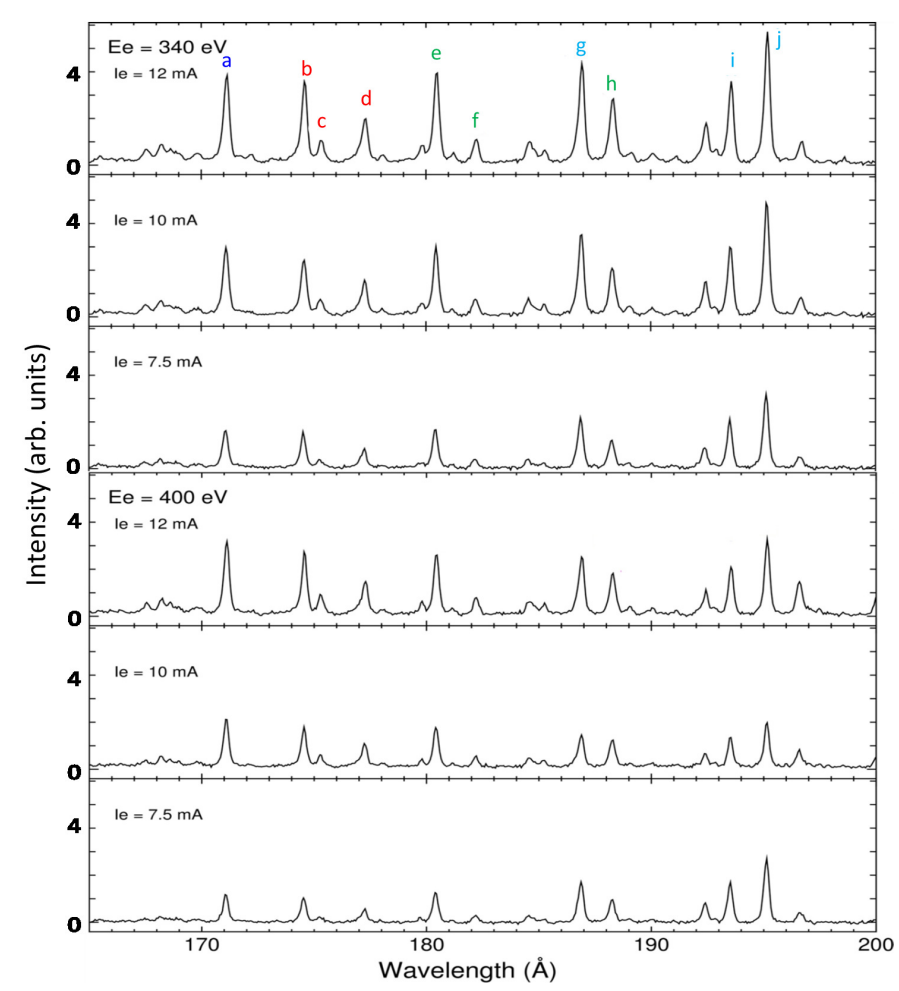

Fig. 3. EUV spectra of highly charged Fe ions recorded with a flat-field grazing incidence spectrometer. The upper three plots are observed at electron beam energy of $340 \mathrm{eV}$, while the lower three plots are obtained at $400 \mathrm{eV}$. The electron beam current is written on each plot. Each spectrum was recorded for an exposure time of $30 \mathrm{~min}$. In the top panel lines from different charge states of present interest are labelled as discussed in the text.

than the effective electron density under the same conditions. This suggests that it is important to extract effective electron densities from EBIT by direct observations rather than obtaining geometrical electron densities by assuming equal size of the electron beam and ion cloud.

\section{Calculations}

The calculations for density dependent line emissivities of Fe X-XII reported in this paper are performed using a collisional-radiative (CR) models. This model has been employed successfully in several previous EBIT studies to identify, simulate and extract spectral line intensities as described in (Nakamura et al. 2011; Ralchenko 2013; Ali et al. 2015; Ding et al. 2016). The CR models give fractional population of ions in the excited states by assuming isotropic and optically thin plasmas as it exists in CoBIT. The state population density at given electron energies and densities can be obtained by solving quasi-stationary state rate equations for the fractional population of each charge state $q, n_{i}^{(q)}$,

$0=\sum_{j}\left(A_{i j}+n_{e} C_{i j}\right) n_{j}^{(q)}-\left(\sum_{j} A_{j i}+n_{e} C_{j i}\right) n_{i}^{(q)}-n_{e} S_{i} n_{i}^{(q)}$,

where $A_{i j}$ and $C_{i j}$ stand for a spontaneous emission rate and electron collision rate coefficients, respectively from the upper level $j$ to the lower level $i$, and $S_{i}$ represents ionization rate coefficients from the level $S_{i}$. The electric-dipole, -quadruple, and -octupole, and those of magnetic-dipole and -quadruple spontaneous transitions are taken into account in the present model calculations. By assuming the delta function of electron energy for the beam energy distribution in the CoBIT, the collision rate coefficients and the ionization rate coefficients are obtained from electron-impact excitation and ionization cross sections, respectively.

The HULLAC code (Bar-Shalom et al. 2001) was used to obtain the atomic data such as energy levels, cross sections of collisional (de)excitation, radiative transition rates to construct the CR model for Fe X-XII ions. Emission from other atomic processes such as radiative, dielectronic and tri-electronic (three body) recombination is expected to have negligible contribution in the present CoBIT spectra because the measurements were performed at electron beam energies below the ionization threshold of each charge state; data from such process is therefore not taken into account while performing CR calculations. The electronic configurations of $3 \mathrm{~s}^{2} 3 \mathrm{p}^{4}(3 \mathrm{~d}, n l), 3 \mathrm{~s} 3 \mathrm{p}^{6}$, and $3 \mathrm{~s} 3 \mathrm{p}^{5}(3 \mathrm{~d}, n l)$ for the excited states of Fe X, $3 \mathrm{~s}^{2} 3 \mathrm{p}^{3}(3 \mathrm{~d}, n l)$, $3 \mathrm{~s} 3 \mathrm{p}^{5}$, and $3 \mathrm{~s} 3 \mathrm{p}^{4}(3 \mathrm{~d}, n l)$ for Fe XI and $3 \mathrm{~s}^{2} 3 \mathrm{p}^{2}(3 \mathrm{~d}, n l), 3 \mathrm{~s} 3 \mathrm{p}^{4}$, and $3 \mathrm{~s}^{3} \mathrm{p}^{3}(3 \mathrm{~d}, n l)$ for those of Fe XII are included, where $n=4,5$ and $l \leq n-1$. Additional electronic configurations that differ by two electrons from $3 \mathrm{~s}^{2} 3 \mathrm{Pk}-13 \mathrm{~d}$ (where $k=5,4$ and 3 for Fe X, Fe XI and Fe XII, respectively) but with the same parity, are also included. Inclusion of this augmentation significantly improved the wavelengths of the spectral lines of present interest. For Fe $\mathrm{X}$, the ground state, $3 \mathrm{~s}^{2} 3 \mathrm{p}^{5}$, is also augmented by including $3 \mathrm{~s}^{2} 3 \mathrm{p}^{3} 3 \mathrm{~d}^{2}$.

\section{Results and discussion}

Figure 3 shows typical data plots recorded with three different electron beam currents at each electron beam energy of 340 and $400 \mathrm{eV}$. These energies are high enough to produce iron ions of present interest. In the wavelength range investigated here the spectral emission is dominated by Fe X, Fe XI and Fe XII as designated with alphabet from $b$ to $j$ in Fig. 3. One strong line at 171.073 A labelled with $a$ is identified as stemming from Fe IX charge state. Higher charge states ( $>$ Fe XII) may also present in the trap at present beam energies, emission from those charge states is however dominant at longer wavelength region as investigated in our earlier work (Nakamura et al. 2011).

The intensity ratios obtained from the spectra's of Fe X, Fe XI and Fe XII are plotted as a function of electron density in Figs. $4 \mathrm{a}-\mathrm{c}$, respectively. The experimental data points obtained at electron beam energies of 340 and $400 \mathrm{eV}$ are given as closed and open symbols, respectively (circles and squares). As described in the introduction, rather than obtaining electron density using theoretical estimations, here we have extracted it experimentally. The horizontal axis in Fig. 4 therefore represents measured electron density derived in the present work. Positive horizontal error bars represent maximum electron density at the centre of the Gaussian electron beam and ion cloud, while negative horizontal error bars represent mechanical uncertainty of the pinhole camera and visible spectrometer. It is important to mention here that no sensitivity corrections has been done for the investigated lines since these lines are close enough to show approximately same sensitivity for the spectrometer and same efficiency of the CCD used in the measurements. In the following subsections, we describe results for density dependence intensity ratios for each charge state in detail. 
Table 1. Charge states and lines investigated in the current work.

\begin{tabular}{ccccc}
\hline \hline Ion & Label & Wavelength $[\AA]$ & Transitions & Possible blending \\
\hline Fe IX & $\mathrm{a}$ & 171.073 & $3 \mathrm{~s}^{2} 3 \mathrm{p}^{6}{ }^{1} \mathrm{~S}_{0}-3 \mathrm{~s}^{2} 3 \mathrm{p}^{5} 3 \mathrm{~d}^{1} \mathrm{P}_{1}$ & \\
Fe X & $\mathrm{b}$ & 174.531 & $3 \mathrm{~s}^{2} 3 \mathrm{p}^{5} \mathrm{P}_{3 / 2}-3 \mathrm{~s}^{2} 3 \mathrm{p}^{4}\left({ }^{3} \mathrm{P}\right) 3 \mathrm{~d}^{2} \mathrm{D}_{5 / 2}$ & \\
& $\mathrm{c}$ & 175.263 & $3 \mathrm{~s}^{2} 3 \mathrm{p}^{5} \mathrm{P}_{1 / 2}-3 \mathrm{~s}^{2} 3 \mathrm{p}^{4}\left({ }^{3} \mathrm{P}\right) 3 \mathrm{~d}^{2} \mathrm{D}_{3 / 2}$ & $\lambda 175.475$ \\
\multirow{5}{*}{ Fe XI } & $\mathrm{d}$ & 177.243 & $3 \mathrm{~s}^{2} 3 \mathrm{p}^{5}{ }^{2} \mathrm{P}_{3 / 2}-3 \mathrm{~s}^{2} 3 \mathrm{p}^{4}\left({ }^{3} \mathrm{P}\right) 3 \mathrm{~d}^{2} \mathrm{P}_{3 / 2}$ & \\
& $\mathrm{e}$ & 180.401 & $3 s^{2} 3 p^{4}{ }^{3} \mathrm{P}_{2}-3 \mathrm{~s}^{2} 3 \mathrm{p}^{3}\left({ }^{4} \mathrm{~S}\right) 3 \mathrm{~d}^{3} \mathrm{D}_{3}$ & $\mathrm{Fe} \mathrm{X} \lambda 180.441$ \\
& $\mathrm{f}$ & 182.167 & $3 s^{2} 3 p^{4}{ }^{3} \mathrm{P}_{1}-3 \mathrm{~s}^{2} 3 \mathrm{p}^{3}\left({ }^{4} \mathrm{~S}\right) 3 \mathrm{~d}^{3} \mathrm{D}_{2}$ & \\
Fe XII & $\mathrm{h}$ & 188.216 & $3 s^{2} 3 p^{4}{ }^{3} \mathrm{P}_{2}-3 \mathrm{~s}^{2} 3 \mathrm{p}^{3}\left({ }^{2} \mathrm{D}\right) 3 \mathrm{~d}^{3} \mathrm{P}_{2}$ & $\lambda 188.089, \lambda 188.299$ \\
& $\mathrm{~g}$ & 186.887 & $3 \mathrm{~s}^{2} 3 \mathrm{p}^{3}{ }^{2} \mathrm{D}_{5 / 2}-3 \mathrm{~s}^{2} 3 \mathrm{p}^{2}\left({ }^{3} \mathrm{P}\right) 3 \mathrm{~d}^{2} \mathrm{~F}_{7 / 2}$ & $\lambda 186.854$ \\
& $\mathrm{i}$ & 193.509 & $3 \mathrm{~s}^{2} 3 \mathrm{p}^{3}{ }^{4} \mathrm{~S}_{3 / 2}-3 \mathrm{~s}^{2} 3 \mathrm{p}^{2}\left({ }^{3} \mathrm{P}\right) 3 \mathrm{~d}^{4} \mathrm{P}_{3 / 2}$ & \\
& $\mathrm{j}$ & 195.119 & $3 \mathrm{~s}^{2} 3 \mathrm{p}^{3}{ }^{4} \mathrm{~S}_{3 / 2}-3 \mathrm{~s}^{2} 3 \mathrm{p}^{2}\left({ }^{3} \mathrm{P}\right) 3 \mathrm{~d}^{4} \mathrm{P}_{5 / 2}$ & \\
\hline
\end{tabular}

Notes. The wavelengths and transitions are listed according to CHIANTI data base.

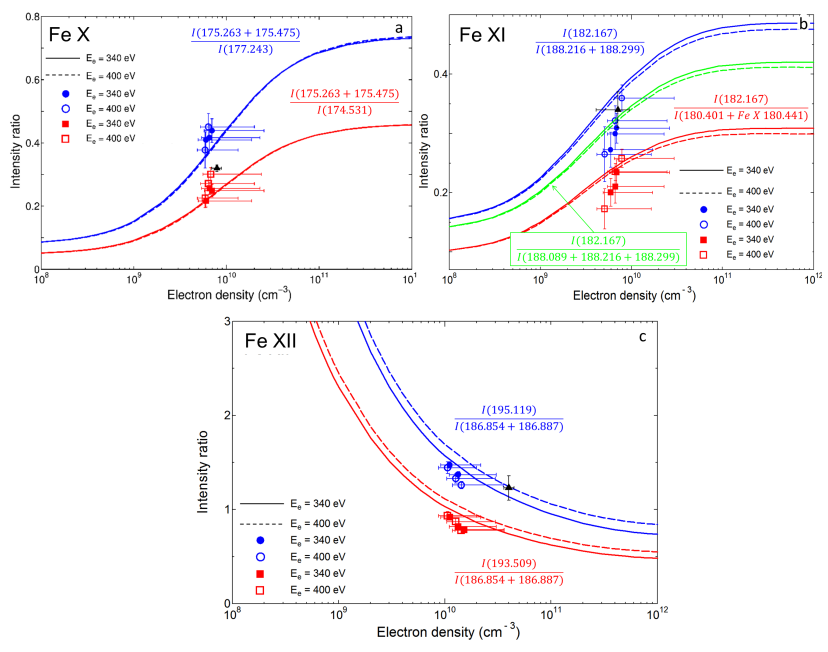

Fig. 4. Comparison of the measured and calculated line intensity ratios for a) $\mathrm{Fe} \quad \mathrm{X}: \quad I(175.263+175.475) / I(177.243)$ and $I(175.263+175.475) / I(174.531)$; b) Fe XI: $I(182.167) / I(188.089+$ $188.216+188.299)$ and $I(182.167) / \quad I(180.401+\mathrm{Fe} \quad \mathrm{X} \quad 180.441)$; and c) Fe XII: $I(195.119) / I(186.854+186.887)$ and $I(193.509) /$ $I(186.854+186.887)$. The vertical error bars were estimated from fitting the Gaussian peak profiles $(1 \sigma)$. Positive horizontal error bars represents maximum electron density at the centre of the Gaussian electron beam and ion cloud, while negative horizontal error bars represents mechanical uncertainty (pinhole camera and visible spectrometer). The symbols with error bars are the measured intensity ratios for electron beam energies of $340 \mathrm{eV}$ (closed squares and circles) and $400 \mathrm{eV}$ (open squares and circles). The solid and dashed lines in a)-c) are our calculated result for 340 and $400 \mathrm{eV}$, respectively. The filled triangles with error bars represents FLASH-EBIT data taken from Liang et al. (2009a). Typical values of the geometrical electron beam densities found in the present work ranges from 2.6 to $4.3 \times 10^{10} \mathrm{~cm}^{-3}$.

\subsection{Fe X}

The emission lines from $\mathrm{Fe} \mathrm{X}$ have been widely observed from the active region and solar flares (Dere et al. 1979; Foster et al. 1996). Several of these lines show density diagnostics potential and are useful to determine the electron density in the solar corona as discussed in several previous publications (Bhatia et al. 1999; Nussbaumer 1976). We identify three strong $\mathrm{Fe} X$ lines in our experimental spectrum, which are labelled as $b, c$ and $d$ in Fig. 3. These correspond to transition $3 \mathrm{~s}^{2} 3 \mathrm{p}^{5}{ }^{2} \mathrm{P}_{3 / 2}-3 \mathrm{~s}^{2} 3 \mathrm{p}^{4}\left({ }^{3} \mathrm{P}\right) 3 \mathrm{~d}{ }^{2} \mathrm{D}_{5 / 2}$ at $\lambda=$ $174.531 \AA, 3 \mathrm{~s}^{2} 3 \mathrm{p}^{5}{ }^{2} \mathrm{P}_{1 / 2}-3 \mathrm{~s}^{2} 3 \mathrm{p}^{4}\left({ }^{3} \mathrm{P}\right) 3 \mathrm{~d}{ }^{2} \mathrm{D}_{3 / 2}$ transition at $\lambda=$ $175.263 \AA$ and $3 \mathrm{~s}^{2} 3 \mathrm{p}^{5}{ }^{2} \mathrm{P}_{3 / 2}-3 \mathrm{~s}^{2} 3 \mathrm{p}^{4}\left({ }^{3} \mathrm{P}\right) 3 \mathrm{~d}^{2} \mathrm{P}_{3 / 2}$ transition at $\lambda=177.243 \AA$ as listed in Table 1 . The following two line pairs $I(175.263) / I(174.531)$ and $I(175.263) / I(177.243)$ are considered to be strongly density sensitive between $N_{\mathrm{e}}=10^{8}$ and $10^{11} \mathrm{~cm}^{-3}$ as discussed by Young et al. (1996) and Keenan et al. (2008).

In moderate solar conditions the $174.531 \AA$ line is quite strong and its intensity is comparable to the well known Fe IX transition at $171.07 \AA$ (Schmitt et al. 1996). This line is one of the strongest lines observed in Procyon and $\alpha$ Cen spectra and does not suffer from any kind of blend or overlap (Foster et al. 1996). In fact no known iron lines exist near this transition either from $\mathrm{Fe} \mathrm{X}$ or other neighbouring charge states. The three lines which may be present in the vicinity of this transition are from O V at $\lambda=172.94,173.08$, and $173.09 \AA$. Firstly, they are not present in our spectra and secondly they are not expected to contribute to this line as they are far away from this transition.

The line at $\lambda=175.263 \AA$ lies in the vicinity of another Fe $\mathrm{X}$ transition $3 \mathrm{~s}^{2} 3 \mathrm{p}^{5} \quad{ }^{2} \mathrm{P}_{3 / 2}-3 \mathrm{~s}^{2} 3 \mathrm{p}^{4}\left({ }^{3} \mathrm{P}\right) 3 \mathrm{~d}{ }^{2} \mathrm{P}_{1 / 2}$ at $175.475 \AA$ and are not resolved with the given resolution of our instrument. This line (Fe X:175.475 $\AA$ ) is well observed and resolved in the SERTS spectra collected with a high-resolution spectrometer, and identified by Malinovsky \& Heroux (1973) and Behring et al. (1976). The contribution of this line is however very small - of the order of $10 \%$ as discussed by Foster et al. (1996) who considered the emissivities calculated by Brickhouse et al. (1995). We also found similar line blending contribution of 175.475 to $175.263 \AA$ in our present calculations. Keenan et al. (2008) also discussed the possibility of intensity contribution to $175.263 \AA$ and concluded that this transition is free from any significant contamination or blending problem. The third Fe X line observed in our spectra at $\lambda=177.243 \AA$ is stronger than $175.263 \AA$ but weaker than $174.531 \AA$. This line is also free from any kind of blending and provides potentially excellent density diagnostics.

The experimental line intensity ratios for $I(175.263+$ $175.475) / I(177.243)$ and $I(175.263+175.475) / I(174.531)$ are plotted as a function of electron density in Fig. 4a. The calculated results are also shown for 340 and $400 \mathrm{eV}$. It can be seen from Fig. 4a, that energy dependence is very small. The calculated intensity ratios fall between the measured data points and hence a good agreement can be seen between both the results. The FLASH-EBIT results from Liang et al. (2009a) for $I(175.263+175.475) / I(174.531)$ is also given as a filled triangle. The result seems to be consistent with our density values, although that need not be necessarily true for all the ratios because of different experimental conditions and EBIT parameters (Nakamura et al. 2011). 


\subsection{Fe XI}

Fe XI emits many strong and bright lines from coronal plasma in EIS wavelength range and not only provides density diagnostics for solar plasma but also useful for instrument calibration (Del Zanna et al. 2010). The complex nature of this ion makes it difficult to identify its energy levels and emission lines. Most of them are still uncertain (Young et al. 2007; Del Zanna et al. 2010) and required further spectroscopic studies as discussed by Keenan et al. (2005). We observed three strong lines of Fe XI in the present CoBIT measurements and labelled as e, $f$ and $h$ in Fig. 3. These appeared at $\lambda=180.401$, 182.167 and $188.216 \AA$ corresponding to transitions from $3 s^{2} 3 p^{4}{ }^{3} P_{2}-3 s^{2} 3 p^{3}\left({ }^{4} S\right) 3 d{ }^{3} D_{3}, 3 s^{2} 3 p^{4}{ }^{3} P_{1}-3 s^{2} 3 p^{3}\left({ }^{4} S\right) 3 d{ }^{3} D_{2}$ and $3 s^{2} 3 p^{4}{ }^{3} P_{2}-3 s^{2} 3 p^{3}\left({ }^{2} \mathrm{D}\right) 3 d^{3} \mathrm{P}_{2}$, respectively. The line ratios $I(182.167) / I(180.401)$ and $I(182.167) / I(188.216)$ are very sensitive to the variation of density but one must take into account the blending effects from neighbouring charge states before using them for density diagnostics as discussed by Pinfield et al. (2001).

The transition at $\lambda=180.401 \AA$ lies in the vicinity of other three lines, two of them at $\lambda=179.758 \AA\left(3 \mathrm{~s}^{2} 3 \mathrm{p}^{4}{ }^{1} \mathrm{D}_{2}-\right.$ $\left.3 \mathrm{~s}^{2} 3 \mathrm{p}^{3}\left({ }^{2} \mathrm{D}\right) 3 \mathrm{~d} \quad{ }^{1} \mathrm{~F}_{3}\right)$ and $180.594 \quad \AA \quad\left(3 \mathrm{~s}^{2} 3 \mathrm{p}^{4} \quad{ }^{3} \mathrm{P}_{1}-\right.$ $\left.3 s^{2} 3 p^{3}\left({ }^{4} S\right) 3 d{ }^{3} D_{1}\right)$ belongs to Fe XI while the third line according to CHIANTI listed at $\lambda=180.441 \AA\left(3 \mathrm{~s}^{2} 3 \mathrm{p}^{5}{ }^{2} \mathrm{P}_{1 / 2}-\right.$ $\left.3 s^{2} 3 p^{4}\left({ }^{3} \mathrm{P}\right) 3 d^{2} \mathrm{P}_{1 / 2}\right)$ originate from $\mathrm{Fe} X$. Our spectrometer resolution is $0.4 \AA$, which means that the transition with wavelength $179.758 \AA$ must be separated from the line 180.401 $\AA$ if it exists in our spectrum. But no such emission from this transition is observed in our recorded spectrum, which shows that this line $(179.758 \AA)$ is very weak at the electron density studied here. The non-existence of this line at the present density have also been reported in previous studies (Schmitt et al. 1996; Pinfield et al. 2001).

As for as the second Fe XI line at $\lambda=180.594 \AA$ is concerned, we estimated its intensity contribution to the line $180.401 \AA$ from our calculations and found negligible contamination $(0.05 \%)$ at an electron density of $1.0 \times 10^{10} \mathrm{~cm}^{-3}$. Thus the only contribution the transition $180.401 \AA$ may contains would be from Fe X transition at $\lambda=180.441 \AA$. Schmitt et al. (1996) and Pinfield et al. (2001) suggested that the Fe X contribution to the Fe XI intensity is about $20 \%$ in the electron density range of present interest. By assuming equal populations of $\mathrm{Fe} \mathrm{X}$ and $\mathrm{XI}$ ions in the trap, we estimated contamination of the $\mathrm{Fe} \mathrm{X}$ line to the $\mathrm{Fe} \mathrm{XI}$ line to be $18 \%$ at an electron density of $1.0 \times 10^{10} \mathrm{~cm}^{-3}$. Since it is considered that Fe XI is dominant at the present beam energies, the contamination should be $\sim 10 \%$ or less. In Fig. 4b, we thus plotted intensity being the sum of 180.401 and 180.441 A transitions from Fe XI and Fe X, respectively.

The second line at $\lambda=182.167 \AA$ in our spectrum labelled with $f$ is uncontaminated from any neighbouring charge state as discussed by Pinfield et al. (2001). The third Fe XI line in our spectrum is observed at wavelength $188.216 \AA$ (according to CHIANTI) and is listed at $188.219 \AA$ in NIST data base and also reported by Liang et al. (2009a), although transition $\left(3 s^{2} 3 p^{4}{ }^{3} P_{2}-3 s^{2} 3 p^{3}\left({ }^{2} \mathrm{D}\right) 3 d^{3} P_{2}\right)$ is same as given in CHIANTI data base. According to CHIANTI line list, there are two lines lying close to $188.216 \AA$, one from Fe XI with the wavelength of $188.299 \AA\left(3 \mathrm{~s}^{2} 3 \mathrm{p}^{4}{ }^{3} \mathrm{P}_{2}-3 \mathrm{~s}^{2} 3 \mathrm{p}^{3}\left({ }^{2} \mathrm{D}\right) 3 \mathrm{~d}^{1} \mathrm{P}_{1}\right)$, and another from Fe XII at $\lambda=188.170 \AA\left(3 \mathrm{~s}^{2} 3 \mathrm{p}^{3}{ }^{2} \mathrm{P}_{1 / 2}-3 \mathrm{~s}^{2} 3 \mathrm{p}^{2}\left({ }^{3} \mathrm{P}\right) 3 \mathrm{~d}^{2} \mathrm{D}_{3 / 2}\right)$.
The transition, at $\lambda=188.299 \AA$ is reported as $3 \mathrm{~s}^{2} 3 \mathrm{p}^{4}{ }^{3} \mathrm{P}_{2}-$ $3 s^{2} 3 p^{3}\left({ }^{2} \mathrm{D}\right) 3 d^{1} \mathrm{P}_{1}$ by Pinfield et al. (2001) and Dere et al. (1997) and also listed in CHIANTI data base, however according to Jupen et al. (1993), Keenan et al. (2005) and Liang et al. (2009a) this line is due to the transition $3 s^{2} 3 p^{4}{ }^{3} P_{2}-3 s^{2} 3 p^{3}\left({ }^{2} D\right) 3 d^{3} S_{1}$. Dere et al. preferred the ${ }^{3} \mathrm{P}_{2}-{ }^{1} \mathrm{P}_{1}$ transition over the Jupe'n et al. identification ${ }^{3} \mathrm{P}_{2}-{ }^{3} \mathrm{~S}_{1}$ due to the weak intensity of the later. This is further studied by Keenan et al. (2005) who found that their calculated intensity of ${ }^{3} \mathrm{P}_{2}-{ }^{3} \mathrm{~S}_{1}$ line is predicted to be $21 \%$ that of the $188.216 \AA$ line, while the ${ }^{3} \mathrm{P}_{2}-{ }^{1} \mathrm{P}_{1}$ intensity is $19 \%$ of 188.216 $\AA$. By considering the fact that intensity of ${ }^{3} \mathrm{P}_{2}-{ }^{3} \mathrm{~S}_{1}$ is slightly larger than ${ }^{3} \mathrm{P}_{2}-{ }^{1} \mathrm{P}_{1}$, Keenan et al. classified this transition as given by Jupen et al. as $3 s^{2} 3 p^{4}{ }^{3} P_{2}-3 s^{2} 3 p^{3}\left({ }^{2} D\right) 3 d^{3} S_{1}$.

The Fe XII transition $\left(3 \mathrm{~s}^{2} 3 \mathrm{p}^{3}{ }^{2} \mathrm{P}_{1 / 2}-3 \mathrm{~s}^{2} 3 \mathrm{p}^{2}\left({ }^{3} \mathrm{P}\right) 3 \mathrm{~d}{ }^{2} \mathrm{D}_{3 / 2}\right)$ at $\lambda=188.170 \AA$ is more than one order of magnitude fainter than the transition at $188.216 \AA$ in the electron density range of present investigation (Schmitt et al. 1996) and must be resolved with the present instrument resolution if it exist in our recorded spectrum. Thus the main contribution may come from the $188.299 \AA$ line. We therefore plotted calculated intensity ratios by adding $188.216 \AA$ and $188.299 \AA$ as shown in Fig. $4 \mathrm{~b}$.

A third line at $\lambda=188.089 \AA\left(3 \mathrm{~s}^{2} 3 \mathrm{p}^{4}{ }^{3} \mathrm{P}_{2}-3 \mathrm{~s}^{2} 3 \mathrm{p}^{3}\left({ }^{2} \mathrm{D}\right) 3 \mathrm{~d}^{3} \mathrm{P}_{1}\right)$ exists in the vicinity of $188.216 \AA$ and may also contribute to the line intensity (Liang et al. 2009a). In order to check its contribution, we plotted calculated values by adding intensity of $188.089,188.216$ and $188.299 \AA$ as shown with the green lines in Fig. 4b. It can be seen from Fig. 4b, that calculated values are higher than the experimental data points when plotted by taking the sum of intensities of the two lines (188.216 and $188.299 \AA$ ) in the denominator. However a good agreement between both the results can be observed when the calculated data is plotted by taking into account the contribution of the three lines (188.089, 188.216 and $188.299 \AA$ ). A previous experimental line intensity ratio from Liang et al. (2009a) for $I(182.167) / I(188.089+188.216+188.299)$ is also shown in the figure and found to be in agreement with our results.

\subsection{Fe XII}

The density diagnostic potential of Fe XII emission lines in the wavelength range of 186-220 $\AA$ has been recognised for many years (Flower 1977; Dere et al. 1979; Vernazza \& Reeves 1978; Kastner \& Mason 1978). We observed three strong Fe XII lines in our experimental spectrum, labelled with $g$, $i$, and $j$ in Fig. 3. These appeared at wavelength $\lambda=186.887$, 193.509, and $195.119 \AA$, corresponding to transitions $3 \mathrm{~s}^{2} 3 \mathrm{p}^{3}{ }^{2} \mathrm{D}_{5 / 2}-$ $3 \mathrm{~s}^{2} 3 \mathrm{p}^{2}\left({ }^{3} \mathrm{P}\right) 3 \mathrm{~d} \quad{ }^{2} \mathrm{~F}_{7 / 2}, \quad 3 \mathrm{~s}^{2} 3 \mathrm{p}^{3}{ }^{4} \mathrm{~S}_{3 / 2}-3 \mathrm{~s}^{2} 3 \mathrm{p}^{2}\left({ }^{3} \mathrm{P}\right) 3 \mathrm{~d} \quad{ }^{4} \mathrm{P}_{3 / 2}$, and $3 s^{2} 3 p^{3}{ }^{4} S_{3 / 2}-3 s^{2} 3 p^{2}\left({ }^{3} P\right) 3 d{ }^{4} P_{5 / 2}$, respectively. All three lines are well observed by EIS (Del Zanna 2012; Storey et al. 2005) and also found in the Procyon spectrum observed with the extreme ultraviolet explorer satellite (Schmitt et al. 1996). The lines at $\lambda=193.509$ and $195.119 \AA$ are quite strong in any of the EIS observations, with $195.119 \AA$ as strongest one lying at the peak of the EIS sensitivity curve (Young et al. 2007). Both lines have almost same density dependence and therefore do not provide any information about the density diagnostics (Brickhouse et al. 1995; Brosius et al. 1998). However, these lines found to be useful for density diagnostics in a wide range of densities $\left(10^{8}-10^{12} \mathrm{~cm}^{-3}\right)$, when taken as a ratio with the line at $186.887 \AA$ (Young et al. 2007; Schmitt et al. 1996). 
We note that the line emission observed at $\lambda=186.887 \AA$ is self-blended with the Fe XII transition $3 \mathrm{~s}^{2} 3 \mathrm{p}^{3}{ }^{2} \mathrm{D}_{3 / 2}-$ $3 \mathrm{~s}^{2} 3 \mathrm{p}^{2}\left({ }^{3} \mathrm{P}\right) 3 \mathrm{~d}^{2} \mathrm{~F}_{5 / 2}$ at $186.854 \AA$ A. Unfortunately, our instrument resolution was not good enough to resolve these two transitions. Therefore, in Fig. 4c, we compare our experimental intensity ratios with the calculated results by taking the sum of intensities of these two transitions. As for as the second line at $\lambda=193.509 \AA$ is concerned, it is unblended and free from overlap with any other transition and thus can be used as a good density diagnostics line relative to $186.887 \AA$ (Young et al. 2009).

The third line at $\lambda=195.119 \AA$ in the recorded spectra may be contaminated with another Fe XII transition $3 s^{2} 3 \mathrm{p}^{3}{ }^{2} \mathrm{D}_{3 / 2}-$ $3 \mathrm{~s}^{2} 3 \mathrm{p}^{2}\left({ }^{1} \mathrm{D}\right) 3 \mathrm{~d}^{2} \mathrm{D}_{3 / 2}$ at $\lambda=195.179 \AA$. According to CHIANTI, the intensity contribution of $195.179 \AA$ line to the $195.119 \AA$ line is predicted to be $\leq 10 \%$ below electron density of $10^{10} \mathrm{~cm}^{-3}$ (Young et al. 2009). From our calculations, we also estimated the contribution of $195.179 \AA$ to $195.119 \AA$ at electron densities of $1 \times 10^{10} \mathrm{~cm}^{-3}$ and $9 \times 10^{14} \mathrm{~cm}^{-3}$ and found to be $10.4 \%$ and $22.7 \%$, respectively. Since our measured density values are about $10^{10} \mathrm{~cm}^{-3}$ therefore no serious overlap is expected to exist between these two lines in the present observed spectrum.

The experimental and calculated intensity ratios for $I(195.119) / I(186.854+186.887)$ and $I(193.509) / I(186.854+$ 186.887) are plotted as a function of electron density in Fig. 4c. The calculated results fall within the experimental uncertainties and thus show a rather good agreement with the measured data points. The previous experimental intensity ratio of $I(195.119) / I(186.854+186.887)$ from Liang et al. (2009a) is also shown in the figure. The intensity ratio seems to be consistent with our experimental values, but electron density is little higher (but within experimental error bars). This might be due to different EBIT parameters and the estimation of the electron-ion overlap factor.

\section{Summary and conclusions}

We have observed high-resolution EUV spectra from highly charged $\mathrm{Fe}$ ions using an electron beam ion trap equipped with a flat-field grazing incidence spectrometer. The spectra were collected at the electron beam energies of 340 and 400 with the beam currents of 7.5, 10 and $12 \mathrm{~mA}$ at each energy. Density dependent intensity ratios important for astrophysical plasma diagnostics were obtained for several line pairs of Fe X, XI, and XII ions. Collisional radiative model calculations were performed using the HULLAC code to estimate the electron density and intensity ratios. Overall good agreement was found between measured and calculated results. Unlike most of the previous studies where theoretical electron-ion overlap factors have been used to obtain electron densities, here we have derived effective electron densities experimentally by using pinhole camera and visible spectrometer. The electron densities and intensity ratios obtained in this study are thus purely experimental and free from theoretical estimations and errors.

Acknowledgements. We would like to thank the Japan Society for the Promotion of Science (JSPS) for providing financial support for this research work.

\section{References}

Ali, S., Shimizu, E., Sakaue, H. A., et al. 2015, Hyperfine Interactions, 235, 45 Bar-Shalom, A., Klapisch, M., \& Oreg, J. 2001, J. Quant. Spectrosc. Radiat Transf., 71, 169

Beiersdorfer, P., \& Lepson J. K. 2012, ApJS, 201, 28

Beiersdorfer, P., Vogel, D. A., Reed K. J., et al. 1996, Phys. Rev. A, 53, 3974

Beiersdorfer, P., Träbert E., Lepson J. K., Brickhouse, N. S., \& Golub, E. 2014a, ApJ, 788, 25
Beiersdorfer, P., Lepson, J. K., Desai, P., Díaz, F., \& Ishikawa, Y. 2014b, ApJS, 210, 16

Bhatia, A. K., \& Doschek, G. A. 1999, At. Data Nucl. Data Tables, 64, 183 Behring, W. E., Cohen, L., Feldman, U., \& Doschek, G. A. 1976, ApJ, 203, 521 Brickhouse, N. S., Raymond, J. C., \& Smith, B. W. 1995, ApJS, 97, 551 Brosius, J. W., Davila, J. M., \& Thomas, R. J. 1998, ApJ, 199, 255 Brown, C. M., Feldman, U., Seely, J. F., et al. 2008, ApJS, 176, 511 Chen, H., Beiersdorfer, P., Heeter, L. A., et al. 2004, ApJ, 611, 598 Culhane, J., Harra, L. K., James, A. M., et al. 2007, Sol. Phys., 243, 19 Dalgarno, A., \& Layzer, D. 1987, Spectroscopy of Astrophysical Plasmas (Cambridge University Press)

Del Zanna, G. 2012, A\&A, 537, A38

Del Zanna, G., Storey, P. J., \& Mason, H. E. 2010, A\&A 514, A40

Dere, K. P., Mason, H. E., Widing, K. G., \& Bhatia, A. K. 1979, ApJS, 40, 341 Dere, K. P., Landi, E., Mason, H. E., Monsignori-Fossi, B. C., \& Young, P. R. 1997, A\&AS, 125, 149

Ding, X., Liu, J., Koike, F., et al. 2016, Phys. Lett. A, 380, 874

Drake, J. J., Laming, J. M., \& Widing, K. G. 1995, ApJ, 443, 393

Dwivedi, B. N. 1993, Space Sci. Rev., 65, 289

Feldman, U., Landi, E., \& Doschek, G. A. 2008, ApJ, 679, 843

Flower, D. R. 1977, A\&A, 54, 163

Foster, J., Mathioudakis, M., Keenan, F. P., Drake, J. J., \& Widing, K. G. 1996, APJ, 473, 560

Gillaspy, J., Aglitskiy, Y., Bell, E. W., et al. 1995, Phys. Scr., T59, 392

Gu, M. F., Savin, D. W., \& Beiersdorfer, P. 1999, J. Phys. B: At. Mol. Opt. Phys., 32,5371

Gúdel, M., \& Nazé, Y. 2009, A\&ARv, 17, 309

Harrison, R. A., Kent, B. J., Sawyer, E. C., et al. 1995, Metrologia, 32, 647

Henderson, J. R., Beiersdorfer, P., Bennett, C. L., et al. 1990, Phys. Rev. Lett., 65,705

Herrmann, G. 1958, J. Appl. Phys., 29, 127

Hu, Z., Li, Y., Han, X., et al. 2014a, Phys. Rev. A, 90, 062702

Hu, Z., Han, X., Li, Y., et al. 2014b, Phys. Rev. Lett., 108, 073002

Hurwitz, M., Sasseen, T. P., \& Sirk, M. M. 2005, ApJ, 623, 911

Jupen, C., Isler, R. C., \& Trabert, E. 1993, MNRAS, 264, 627

Kastner, S. O., \& Mason, H. E. 1978, A\&A, 67, 119

Keenan, F. P., Aggarwal, K. M., Ryans, R. S. I., et al. 2005, ApJ, 624, 428

Keenan, F. P., Jess, D. B., Aggarwal, K. M., et al. 2008, MNRAS, 389, 939

Kurth, W. S., De Pascuale, S., Faden, J. B., et al. 2015, J. Geophys. Res. Space Phys., 120, 904

Liang, G. Y., Baumann, T. M., Crespo López-Urrutia, J. R., et al. 2009a, ApJ, 696, 2275

Liang, G. Y., Crespo López-Urrutia, J. R., Baumann, T. M., et al. 2009b, ApJ, 702,838

Lemen, J. R., Akin, D. J., Boerner, P. F., et al. 2012, Sol. Phys., 275, 17

Lepson, J. K., Beiersdorfer, P., Hurwitz, M., et al. 2008, J. Phys. Conf. Ser., 130, 012014

Malinovsky, M., \& Heroux, L. 1973, ApJ, 181, 1009

Mason, H. E., Young, P. R., Pike, C. D., et al. 1997, Sol. Phys., 170, 143

Mewe, R., Raassen, A. J. J., Drake, J. J., et al. 2001, A\&A, 368, 888

Nakamura, N., Kikuchi, H., Sakaue, H. A., \& Watanabe, T. 2008, Rev. Sci. Instrum., 79, 063104

Nakamura, N., Watanabe, E., Sakaue, H. A., et al. 2011, ApJ, 739, 17

Neupert, W. M., Epstein, G. L., Thomas, R. J., \& Thompson, W. T. 1992, Sol. Phys., 137, 87

Nussbaumer, H. 1976, A\&A, 48, 93

Pinfield, D. J., Keenan, F. P., \& Mathioudakis, M., et al. 2001, ApJ, 562, 566

Ralchenko, Y. 2013, Plasma Fusion Res., 8, 2503024

Sakaue, H. A., Nakamura, N., Watanabe, E., Komatsu, A., \& Watanabe, T. 2010, J. Instrum., 5, C08010

Schmitt, J. H. M. M., Drake, J. J., Haisch, B. M., \& Stern, R. A. 1996, ApJ, 467, 841

Shah, C., Jörg, H., Bernitt, S., et al. 2015, Phys. Rev. A, 92, 042702

Shestov, S. V., Urnov, A. M., Kuzin, S. V., Zhitnik, I. A., \& Bogachev, S. A. 2009, Astron. Lett., 35, 45

Silver, E., Schnopper, H., Bandler, S., et al. 2000, ApJ, 541, 495

Storey, P. J., Del Zanna, G., Mason, H. E., \& Zeippen, C. J. 2005, A\&A, 433, 717

Taḱacs, E., Meyer, E. S., Gillaspy, J. D., et al. 1996, Phys. Rev. A, 54, 1342

Thomas, R. J., \& Neupert, W. M. 1994, ApJS, 91, S461

Vernazza, J. E., \& Reeves, E. M. 1978, ApJS, 37, 485

Wamsteker, W., Prochaska, J. X., Bianchi, L., et al. 2006, Ap\&SS, 303, 69

Yamamoto, N., Kato, T., Funaba, H., et al. 2008, ApJ, 689, 646

Young, P. R., Mason, H. E., Bhatia, A.K., Doschek, G. A., \& Thomas, R. J. 1996, in Astrophysics in the Extreme Ultraviolet (Kluwer Academic Publishers), IAU Symp., 152

Young, P. R., Del Zanna, G., Mason, H. E., et al. 2007, PASJ, 59, S857

Young, P. R., Watanabe, T., Hara, H., \& Mariska, J. T. 2009, A\&A, 495, 587 